\title{
INDICE GENERALE DELL'ANNATA 2020
}

\section{2}

\section{Gennaio-Giugno, N. 1}

Guido Montani - Ideology, utopia and the crisis of

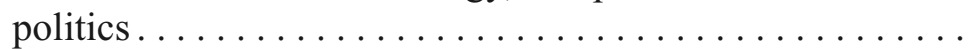

Francesco MAZZUCOTELLI - Fragments of Lebanon: Sectarianism and the Financial Crisis . . . . . . . . . . . . . .

DOMENico Moro - Difesa europea e nuovo ordine mondiale ...........................

The European Central Bank's Communication at the times of Mario Draghi

JACQUeS ZILleR - The European Central Bank's Communication at the Times of Mario Draghi ..........

Allegra Canepa - Unconventional Monetary Policies and Communication Strategies of the European Central Bank . .

Thierry Bracke - Central Bank Communication in the Past Two Decades. The ECB's Experience. . . . . . . . . . . . . .

Alessandro Giovannini , JeAn-François Jamet - Matching Accountability with Independence: the ECB's Experience . .

\section{Note e discussioni}

Renata Targetti Lenti - Il Keynes di Giorgio La Malfa: una nota 


\section{Recensioni e segnalazioni}

Giancarlo Vilella, Working Methods of the European Parliament Administration in a Multi-actors World. A casestudy (FABIo RugGe) . . . . . . . . . . . . . . . . . .

Carlo Cottarelli, Pachidermi e Pappagalli. Tutte le bufale sull'economia a cui continuiamo a credere (RENATA TARgetti Lenti) ......................

Andrea Lorenzo Capussela, Declino. Una storia italiana (R.T.L.) . . . . . . . . . . . . . . . . . . . . . .

Ferruccio de Bortoli, Salvatore Rossi, La ragione e il buonsenso. Conversazione patriottica sull'Italia (R.T.L.) . .

Guido Montani, Ideologia, economia e politica, Il federalismo sovranazionale come pensiero emergente (R.T.L.) . . . . . . . . . . . . . . . . . . . .

Michele Alacevich, AnNa Soci, Breve storia della disuguaglianza (R.T.L.). . . . . . . . . . . . . . .

Cosma Emilio Orsi, Alle origini del reddito di cittadinanza. Teorie economiche e 'welfare state' dal XVI secolo a oggi (R.T.L.) ................................

P. Martelli, L'istituzione del disordine. Regole del gioco e giocatori nella politica italiana dal 1946 al 2018 (GiAnLUCA DAMIANI).....................

A. Panebianco, Persone e mondi. Azioni individuali e ordine internazionale (G.D.) . . . . . . . . . . . . . . . . 


\section{Luglio-Dicembre, N. 2}

Keith Jakee, Stephen Jones-Young - Social Norms and the Rule of Law: an Application to Failed States . . . . . . . . .

Michele Benazzo - Failed States and Tilly's Theory: a Historical Revision of Functional Failure in the Afghan Case... . . . . . . . . . . . . . . . . . . . . . . . . .

\section{Per i Settanta anni de "Il Politico"}

Raimondo Cubeddu - Leoni and Hayek on Nomos and

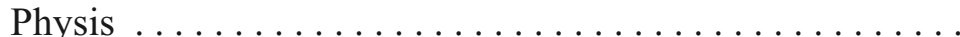

Marco Clementi - "Il Politico" e le Relazioni internazionali.

Note e discussioni

Claudio Giulio AnTA - Idee e modelli del pacifismo tra XIX e XX secolo . . . . . . . . . . . . . . . . . . . .

IlARIA PIZZA - La dinamica dei doveri e le forme della giustizia nel modello politico del giovane Grozio . . . . . . .

GiUlio Di DonATo - Leopardi politico tra amor patrio e fratellanza universale.

Silvia FAVALLI - Misure di austerità e discriminazione sulla base della disabilità: una recente decisione della corte di Strasburgo ........................... 


\section{Recensioni e segnalazioni}

NADia Urbinati, Thinking Democracy Now. Between Innovation and Regression (RENATA TARGETTI LENTI). . . . . .

Carola Ricci, Building an Inclusive Digital Society for Persons with Disabilities. New Challenges and Future Potentials (DeLIA Ferri) . . . . . . . . . . . . . . . . . . .

RAINER ZitelmanN, La forza del capitalismo. Un viaggio nella storia recente dei cinque continenti (CARlo Marsonet), . . . . . . . . . . . . . . .

Angelo Panebianco, Sergio Belardinelli, All'alba di un nuovo mondo (C.M.) . . . . . . . . . . . . . . . . . .

Giuseppe Celi, Andrea Ginzburg, Dario Guarascio, ANNAMARIA SimONAZZI, Una Unione divisiva. Una prospettiva centro-periferia della crisi europea

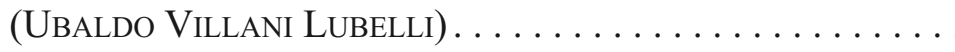

